Original Articles

\title{
Oncogenic role of TYRO3 receptor tyrosine kinase in the progression of pancreatic cancer
}

\author{
Masaki Morimoto $^{\mathrm{a}, \mathrm{b}}$, Yosuke Horikoshi ${ }^{\mathrm{a}}$, Kazuhiro Nakaso ${ }^{\mathrm{a}}$, Tatsuyuki Kurashiki ${ }^{\mathrm{a}, \mathrm{c}}$, \\ Yoshinori Kitagawa $^{\mathrm{a}, \mathrm{c}}$, Takehiko Hanaki ${ }^{\mathrm{b}}$, Teruhisa Sakamoto ${ }^{\mathrm{b}}$, Soichiro Honjo ${ }^{\mathrm{b}}$, \\ Yoshihisa Umekita $^{\mathrm{d}}$, Yoshiyuki Fujiwara ${ }^{\mathrm{b}}$, Tatsuya Matsura ${ }^{\mathrm{a}, *}$ \\ ${ }^{a}$ Division of Medical Biochemistry, Department of Pathophysiological and Therapeutic Science, Faculty of Medicine, Tottori University, 86 Nishi-cho, Yonago, 683-8503, \\ Japan \\ ${ }^{\mathrm{b}}$ Division of Surgical Oncology, Department of Surgery, Faculty of Medicine, Tottori University, 36-1 Nishi-cho, Yonago, 683-8504, Japan \\ ${ }^{\mathrm{c}}$ Division of Anesthesiology and Critical Care Medicine, Department of Surgery, Faculty of Medicine, Tottori University, 36-1 Nishi-cho, Yonago, 683-8504, Japan \\ ${ }^{\mathrm{d}}$ Division of Organ Pathology, Department of Pathology, Faculty of Medicine, Tottori University, 86 Nishi-cho, Yonago, 683-8503, Japan
}

\section{A R T I C L E I N F O}

\section{Keywords:}

Akt

ERK

Invasion

Proliferation

Prognosis

TAM family

\begin{abstract}
A B S T R A C T
The expression and functions of TYRO3, a member of the TAM receptor tyrosine kinase family, in pancreatic cancer (PC) have not been specifically elucidated. In this study, we confirmed TYRO3 expression in five human PC cell lines (PANC-1, MIA PaCa-2, BxPC-3, AsPC-1, and PK-9) using Western blotting. TYRO3 silencing and overexpression studies have revealed that TYRO3 promotes cell proliferation and invasion in PC via phosphorylation of protein kinase B (Akt) and extracellular signal-regulated kinase (ERK). Using a mouse xenograft model, we showed that tumor growth was significantly suppressed in mice subcutaneously inoculated with TYRO3-knockdown PC cells compared with mice inoculated with control PC cells. Furthermore, TYRO3 expression was examined in PC tissues obtained from 106 patients who underwent pancreatic resection for invasive ductal carcinoma through immunohistochemical staining. TYRO3-positive patients had poor prognoses for overall survival and disease-specific survival compared with TYRO3-negative patients. Multivariate analysis revealed that TYRO3 expression is an independent prognostic factor for overall survival. Our study demonstrates the critical role of TYRO3 in PC progression through Akt and ERK activation and suggests TYRO3 as a novel promising target for therapeutic strategies against PC.
\end{abstract}

\section{Introduction}

Despite developments in the diagnostic procedure and treatment of pancreatic cancer (PC) [1], patients are associated with poor prognoses ( $<10 \% 5$-year survival) [2]. This is attributed to the limited number of patients diagnosed at an early stage and resistance of PC to chemotherapy. Compared with the conventionally used gemcitabine, a DNA synthesis inhibitor, the newly adopted cytotoxic chemotherapy FOLFIRINOX (a combination of 5-fluorouracil, leucovorin, irinotecan, and oxaliplatin) has improved the overall survival (OS) of patients with PC. However, because the new regimen is highly toxic, tolerability remains a concern [3].
The most frequent genetic abnormalities in invasive pancreatic adenocarcinomas are mutational activation of the Kirsten rat sarcoma viral homolog (K-RAS) oncogene and inactivation of tumor-suppressor genes, including CDKN2A, TP53, SMAD4, and BRCA2 [4]. The receptor tyrosine kinases (RTKs), epidermal growth factor receptor (EGFR), and human epidermal growth factor receptor 2 are concomitantly upregulated with oncogenic K-RAS expression [5]. EGFR upregulation, caused by K-RAS and loss of SMAD4, plays an important role in increased tumor invasion [6]. Recently, RTKs and the downstream signaling pathways involved in PC development and progression have been targeted for the treatment of cancer [7]. Several clinical trials using small molecule inhibitors, such as the selective EGFR tyrosine kinase inhibitor

\footnotetext{
Abbreviations: BrdU, bromodeoxyuridine; EMT, epithelial to mesenchymal transition; GAS6, growth arrest-specific protein 6; OS, overall survival; PC, pancreatic

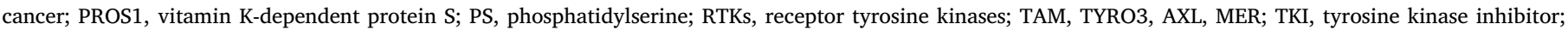

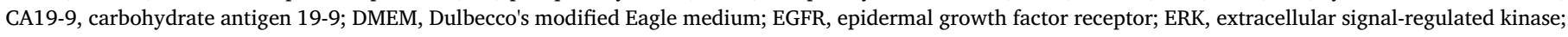
FBS, fetal bovine serum

* Corresponding author.

E-mail address: tmatsura@tottori-u.ac.jp (T. Matsura).
} 
(TKI) erlotinib [8] or the RET/VEGFR/EGFR TKI vandetanib [9], combined with gemcitabine for advanced PC, have been previously performed. These studies have demonstrated limited efficacies or nonsignificant results compared with conventional therapies using solely gemcitabine. In addition to the cell proliferation signals produced by genetic mutations, the tumor microenvironment (i.e., tumor-induced immunosuppression [10] and a certain type of inflammation [11,12]) is involved in PC development. The molecular mechanism involved in PC progression remains to be fully elucidated. Therefore, further investigations to develop therapies targeting the signal cascades of tumor progression in PC are required.

The TYRO3, AXL, and MER members of the TAM family of RTKs were among the latest to evolve [13] and be identified [14-16]. The members of this family exhibit similar domain structures and are highly related through a unique KWIAIES sequence conserved in their kinase domain [17]. TAM activation mechanisms are unique, and they require both an extracellular lipid moiety and a bridging protein ligand for maximal stimulation. The ligands, growth arrest-specific protein 6 (GAS6), and vitamin K-dependent protein S (PROS1) are $\gamma$-carboxylated proteins, which bind to both the receptor and phosphatidylserine (PS) via their carboxyl-terminal and amino-terminal domains, respectively. More recently, other tissue- and receptor-specific ligands, such as tubby and galectin-3, have been identified [18,19]. TAM RTKs are reportedly ectopically induced or overexpressed in various human cancers, and their signals promote survival, chemoresistance, migration, and invasion of PC $[20,21]$. There are differences in the regulation of AXL and MER expressions [17]. Furthermore, the TAM RTKs expressed in individual tumors or in different tumor types are not redundant [17]. In other words, each TAM family member may play a different role in tumor cells. The selective AXL inhibitor BGB324, currently used in phase II clinical trials for multiple types of cancer, may also be a promising drug for treating PC [22]. Regarding MER, the MER-selective inhibitor UNC2025 exhibits antitumor effects in preclinical models of melanoma but not in PC [23]. To date, there is limited data regarding the selective inhibitor of TYRO3 (the third member of the TAM family) in any type of tumor.

Moreover, although it has been reported that TYRO 3 exhibits oncogenicity and has potential as a therapeutic target in colon cancer [24], only few studies have suggested that TYRO3 is a potential oncogene in solid tumors, such as thyroid cancer [25] and melanoma [26], and its function in PC has not been specifically addressed. Given the different regulation and roles of TAM RTKs in tumors, targeting all TAM RTKs activated in individual tumors may lead to a novel strategy for cancer therapy.

In the present in vitro and in vivo studies, we evaluate the oncogenic role of TYRO3 in the proliferation, invasion, and prognosis of PC through growth signal activation.

\section{Materials and methods}

\subsection{Materials}

The PANC-1, BxPC-3, and MIA PaCa-2 cell lines developed from the PC samples and the AsPC-1 cell line derived from the ascites of a patient with metastatic PC were purchased from the American Type Culture Collection (Manassas, VA). PK-9 cells derived from a resected PC biopsy specimen were obtained from the Cell Resource Center of Biomedical Research Institute of Development, Aging and Cancer at Tohoku University. The human pancreatic duct epithelial (HPDE) cell line H6c7 was obtained from Kerafast (Boston, MA). The commercially available antibodies used for Western blotting were as follows: anti-TYRO3 (\#5585; Cell Signaling Technology, Danvers, MA), anti-phospho-Akt (\#4060; Cell Signaling Technology), anti-Akt (\#9272; Cell Signaling Technology), anti-phospho-ERK (\#7383; Santa Cruz Biotechnology, CA), anti-ERK (\#s94; Santa Cruz Biotechnology), anti- $\beta$-actin (\#47778; Santa Cruz Biotechnology), anti- $\mathrm{Na}^{+} / \mathrm{K}^{+}$ATPase (\#14418-1-AP;
Proteintech, Chicago, IL), anti-Lamin B1 (\#66095-1-Ig; Proteintech), and anti-SNAIL (\#3879; Cell Signaling Technology). For immunohisto/ cytochemistry, anti-TYRO3 (\#HPA071245; Sigma-Aldrich, St. Louis, MO), anti-phospho-ERK (\#4370; Cell Signaling Technology), and antiKi-67 (\#418071; Nichirei Bio Science, Tokyo, Japan) were used in addition to the abovementioned anti-phospho-Akt antibody (\#4060). Bromodeoxyuridine (BrdU) was purchased from Tokyo Chemical Industry Co., Ltd. (Tokyo, Japan). The anti-BrdU mouse monoclonal antibody was purchased from Medical \& Biological Laboratories Co., Ltd. (Nagoya, Japan). The PI3K inhibitor LY294002 (LY) was purchased from Alomone Labs (Jerusalem, Israel). The MEK inhibitor PD98059 (PD) was purchased from Alexis (San Diego, CA).

\subsection{Cell culture}

PANC-1 and MIA PaCa-2 cells were cultured in Dulbecco's modified Eagle medium (DMEM; Nissui Pharmaceutical, Tokyo, Japan) and supplemented with $10 \%$ fetal bovine serum (FBS), whereas BxPC-3, AsPC-1, and PK-9 cells were cultured in RPMI 1640 (Nissui Pharmaceutical) supplemented with 10\% FBS. HPDE cells were maintained in Keratinocyte serum-free medium containing supplied supplements (\#17005-042, ThermoFisher Scientific, Waltham, MA).

\subsection{Western blotting}

The methods used for Western blotting have been described previously [27]. The signals were visualized using the Immobilon Western chemiluminescent substrate (Millipore, Billerica, MA) and quantified using the Image Quant LAS 4000 mini (GE Healthcare, Chicago, IL).

\subsection{BrdU incorporation assay}

A total of $1 \times 10^{5}$ cells were seeded into a 24-well plate and incubated at $37{ }^{\circ} \mathrm{C}$ for $24 \mathrm{~h}$. BrdU incorporation was determined after a 2$\mathrm{h}$ pulse, as described previously [28]. For experiments including inhibitors, the cells were pretreated with LY $(20 \mu \mathrm{M})$ or PD $(10 \mu \mathrm{M})$ for $4 \mathrm{~h}$ and analyzed by the BrdU incorporation assay.

\subsection{Cell invasion assay}

Cell invasion was analyzed using a Transwell assay $(8-\mu \mathrm{m}$ pore size; Corning, Corning, NY) as described previously [28]. For experiments including inhibitors, the cells were pretreated with LY $(20 \mu \mathrm{M})$ or PD $(10 \mu \mathrm{M})$ for $24 \mathrm{~h}$ and analyzed by the Transwell assay.

\subsection{Gene silencing of TYRO3 using siRNA}

For siRNA transfection, PANC-1 and MIA PaCa-2 cells $\left(1 \times 10^{6}\right.$ cells) were seeded into 6-well plates with normal medium. In accordance with the manufacturer's protocol, cells were transfected with siTYRO3 reagent (\#36438; Santa Cruz Biotechnology), which is a pool of three different siRNA duplexes (Supplementary Table 1), or control siRNA reagent (\#37007; Santa Cruz Biotechnology) and incubated for $24 \mathrm{~h}$. After replacing the medium, the cells were cultured for $48 \mathrm{~h}$ and analyzed.

\subsection{Establishment of TYRO3-overexpressing PC cells}

For TYRO3 overexpression, PANC-1 cells were plated in 24-well plates $\left(0.5 \times 10^{5} /\right.$ well $)$ and incubated for $24 \mathrm{~h}$ at $37^{\circ} \mathrm{C}$. The full-length cDNA of TYRO3 was inserted into the pEGFP-C2 vector (CLONTECH, Mountain View, CA) with a N-terminal green fluorescent protein tag. The vector was transfected into PANC-1 cells using Lipofectamine LTX (ThermoFisher Scientific, Walthem, MA) according to the manufacturer's protocol, and cells were incubated for $24 \mathrm{~h}$. Subsequently, the cells were passaged at 1:10 dilution into fresh growth medium 
containing G418 $(0.2 \mathrm{mg} / \mathrm{mL})$ and maintained thereafter. After 1-2 weeks, the cells were assayed using Western blotting for TYRO3 expression.

\subsection{Immunofluorescence analysis}

Immunofluorescence was performed using confocal microscopy (LSM780; Carl Zeiss Microscopy, Jena, Germany), as described previously, to examine the intracellular localization of phospho-Akt and phospho-ERK [28].

\subsection{Cell fractionation}

For membrane fractions, cell fractionation was performed as described previously [28]. For nuclear fractions, the cells were harvested, washed, and resuspended in $1 \mathrm{~mL}$ hypotonic buffer $(10 \mathrm{mM}$ Tris-HCl, $\mathrm{pH} 7.4,10 \mathrm{mM} \mathrm{KCl}, 3 \mathrm{mM} \mathrm{MgCl}$ ). After incubation on ice for $15 \mathrm{~min}$, $25 \mu \mathrm{L}$ of $20 \% \mathrm{NP}-40$ was added to the cells, the samples were centrifuged for $10 \mathrm{~min}$ at $650 \mathrm{~g}$, and the supernatants were discarded. The nuclear pellets were resuspended in $50 \mu \mathrm{L}$ cell extraction buffer $(10 \mathrm{mM}$ Tris-HCl, pH 7.4, $100 \mathrm{mM} \mathrm{NaCl}, 2 \mathrm{mM} \mathrm{Na}_{3} \mathrm{VO}_{4}, 1 \%$ Triton X-100, $10 \%$ glycerol, $1 \mathrm{mM}$ EGTA, $0.1 \%$ SDS, $1 \mathrm{mM} \mathrm{NaF}, 20 \mathrm{mM} \mathrm{Na}{ }_{4} \mathrm{P}_{2} \mathrm{O}_{7}$ ) for $30 \mathrm{~min}$ on ice, with vortexing at 10 -min intervals. Finally, the samples were centrifuged for $30 \mathrm{~min}$ at $14,000 \mathrm{~g}$ at $4{ }^{\circ} \mathrm{C}$, and the supernatants were collected as the nuclear fractions.

\subsection{Establishment of stable TYRO3 shRNA-expressing cells}

The lentiviral green fluorescent protein vector, pGFP-C-shLenti, was constructed with four unique 29mer shRNA \#A-D (TL316973; OriGene Technologies, Rockville, MD) (Supplementary Table 2). Twenty-four $\mathrm{h}$ after seeding in a 6-well plate $\left(5.0 \times 10^{6}\right.$ cells/well), Lenti-X 293T cells were transfected with $1 \mu \mathrm{g}$ of shRNA vector plasmid and $1.2 \mu \mathrm{g}$ of the lentiviral packaging plasmid (TR30037; OriGene Technologies). The medium was replaced by fresh DMEM with 10\% FBS at 24 and $48 \mathrm{~h}$ after transfection. The media collected $48 \mathrm{~h}$ after transfection were pooled and filtered $(0.45 \mu \mathrm{m})$. To generate a stable cell line, PANC1 cells were seeded into 24 -well plates $\left(0.5 \times 10^{5}\right.$ /well $)$ and incubated for $18-20 \mathrm{~h}$ until they reached $50 \%$ confluency. After removing the medium, lentiviral particles that were suspended in $0.5 \mathrm{~mL}$ DMEM were added. After $24 \mathrm{~h}$ of incubation, the medium was replaced with medium that did not contain viral particles. Puromycin was used for selecting single-cell colonies.

\subsection{Subcutaneous xenograft models}

Each group comprised six mice (4-6 week-old male BALB/c nu/nu mice; CLEA Japan, Tokyo, Japan) and was subcutaneously inoculated into the right dorsal portion with stable TYRO3 shRNA-expressing cells or control cells $\left(10^{7}\right.$ cells) suspended in $200 \mu \mathrm{L}$ DMEM. The tumor volume was calculated for five weeks after injection using the following formula: length $\times$ width [2] $\times 0.5$ measured using calipers every week. This experimental protocol was approved by the Animal Care Committee of Tottori University (approval number: h30-Y029). All efforts were made to minimize the number of animals involved in this study.

\subsection{Immunohistochemical staining}

Between May 2006 and March 2018, 106 consecutive patients underwent pancreatic resection at Tottori University Hospital (Yonago, Japan) for pancreatic invasive ductal carcinoma; all these patients were included in the analysis. This protocol was approved by the Tottori University Ethical Board (approval number: 17A135), and all patients provided written informed consent for pathological analysis. The clinicopathological parameters and laboratory data of all patients were extracted from their electronic medical records. The patients' characteristics (i.e., age, sex, body mass index, tumor location, and cancer type) and surgical and perioperative parameters (i.e., neoadjuvant chemotherapy, adjuvant chemotherapy, pathological stage, histological type, vascular invasion, neural invasion, $\mathrm{T}$ stage, lympho-node metastasis, surgical margin, carcinoembryonic antigen, and carbohydrate antigen 19-9 [CA19-9]) were retrospectively analyzed.

The 4- $\mu$ m-thick unstained sections from paraffin-embedded blocks were stained with the antibodies and counterstained with hematoxylin to detect nuclei. The cells with strong staining of the cytoplasm and/or plasma membrane were considered positive for TYRO-3 expression. Strong staining was defined as staining intensity equal to that of the islet of Langerhans cells in non-cancerous pancreatic tissues, which served as internal positive controls. For analysis, a two-tiered classification using positive and negative staining was used. Tumors with > $10 \%$ positive cells were classified as TYRO3 positive. Immunolabelling was evaluated by two investigators (Y.U. and M.M.), and consensus was reached in all the cases.

\subsection{Statistical analysis}

All statistical analyses were performed using SPSS Version 23.0 (IBM Corporation, Armonk, NY). P values of $<0.05$ denoted statistical significance. Univariate analyses were performed using Fisher's exact test for categorical variables and two-tailed t-tests for continuous variables. Survival curves were analyzed using the Kaplan-Meier method, and the differences in survival curves were compared using the log-rank test. Cox proportional hazards models were used for the multivariate analysis.

\section{Results}

\subsection{TYRO3 expression in PC and HPDE cell lines}

Although TYRO3 expression has been reported in colon cancer [24], thyroid cancer [25], and melanoma [26], whether PC cells express TYRO3 remains unknown. Therefore, we first examined TYRO3 expression in five human PC cell lines (PANC-1, MIA PaCa-2, BxPC-3, AsPC-1, and PK-9) and in the control HPDE cell line. We found that TYRO-3 was expressed at various levels in all the tested cell lines (Fig. 1A).

\subsection{Silencing of TYRO3 in PC cells suppresses the downstream growth signal and tumor progression in vitro}

TYRO3 is considered a potential oncogene [24-26]; therefore, we performed TYRO3 knockdown using specific siRNA to determine whether TYRO3 is required for tumor progression in PC. The PC cells (PANC-1 and MIA PaCa-2) expressing TYRO3 siRNA displayed a significant reduction in TYRO3 protein expression (Fig. 1B). Subsequently, we examined the effect of TYRO3 knockdown on cell proliferation in PANC-1 and MIA PaCa-2 cells. Incorporating BrdU in the cells after a 2$\mathrm{h}$ pulse assessed cell proliferation. Depleting TYRO3 significantly suppressed BrdU incorporation compared with the control (Figs. 1C and 1D). In addition, we performed the Transwell assay to explore the effects of TYRO3 knockdown on cell invasion. The results showed that TYRO3 silencing significantly reduced the invasive abilities of PC cells (Figs. 1E and 1F). These results clearly indicate that TYRO3 is necessary for PC progression, including cell proliferation and invasion.

Regarding TAM receptor signaling in human cancers, GAS6 stimulation activates PI3K/Akt signaling in multiple cancers, following the interaction of GAS6 with AXL or MER [21,29,30]. However, downstream PI3K/Akt activation of TYRO3 engagement has not been extensively characterized. Therefore, we analyzed Akt phosphorylation in PANC-1 and MIA PaCa-2 cells after TYRO3 silencing to investigate whether the PI3K/Akt signal pathway is activated downstream of 
A

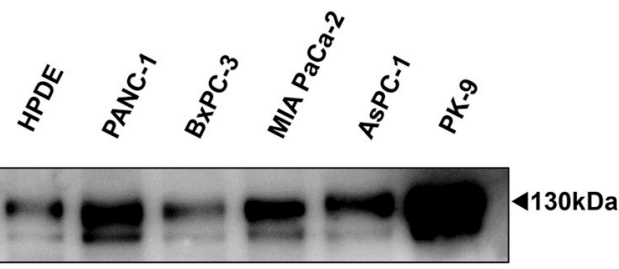

$\beta$-actin

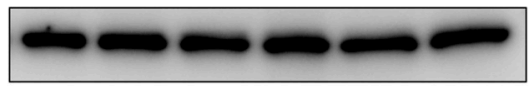

B

PANC-1

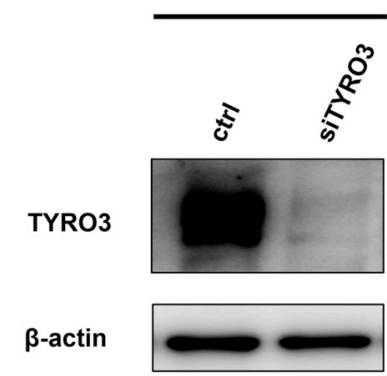

PANC-1

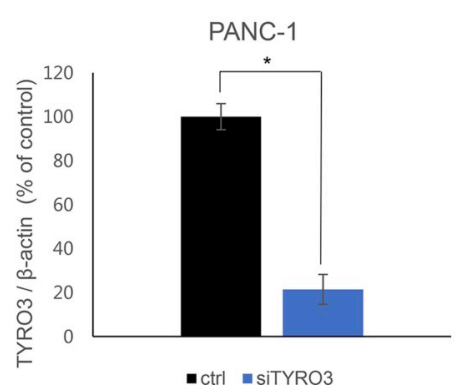

MIA PaCa-2
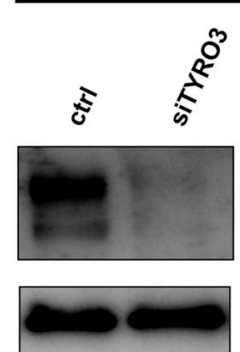

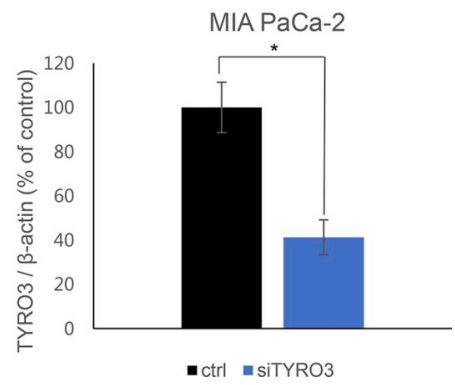

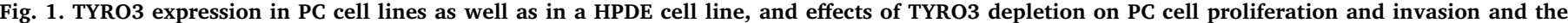

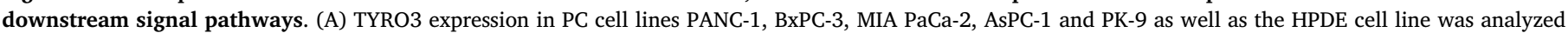

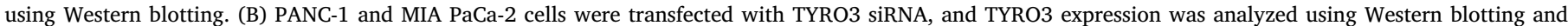

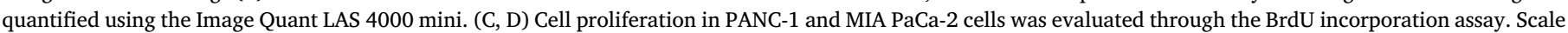

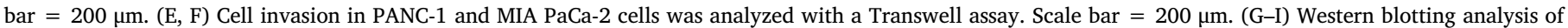

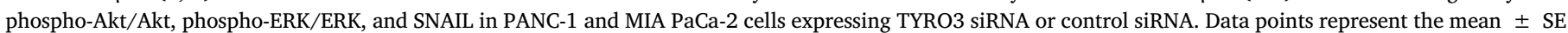
of three independent experiments. ${ }^{*} \mathrm{P}<0.05$. ctrl, control.

C
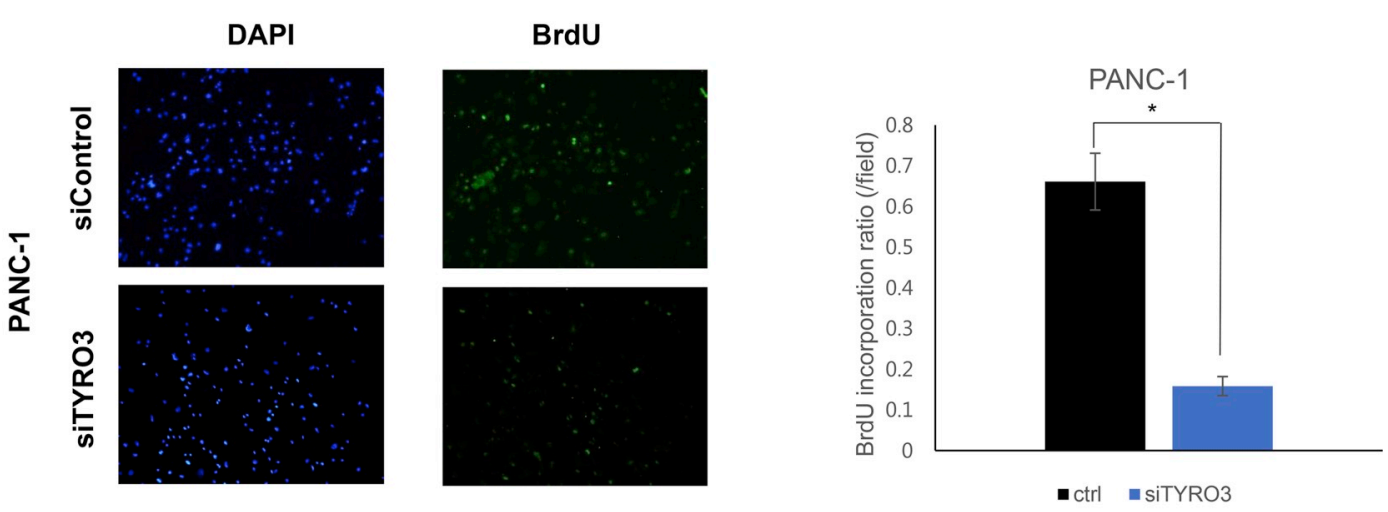

D
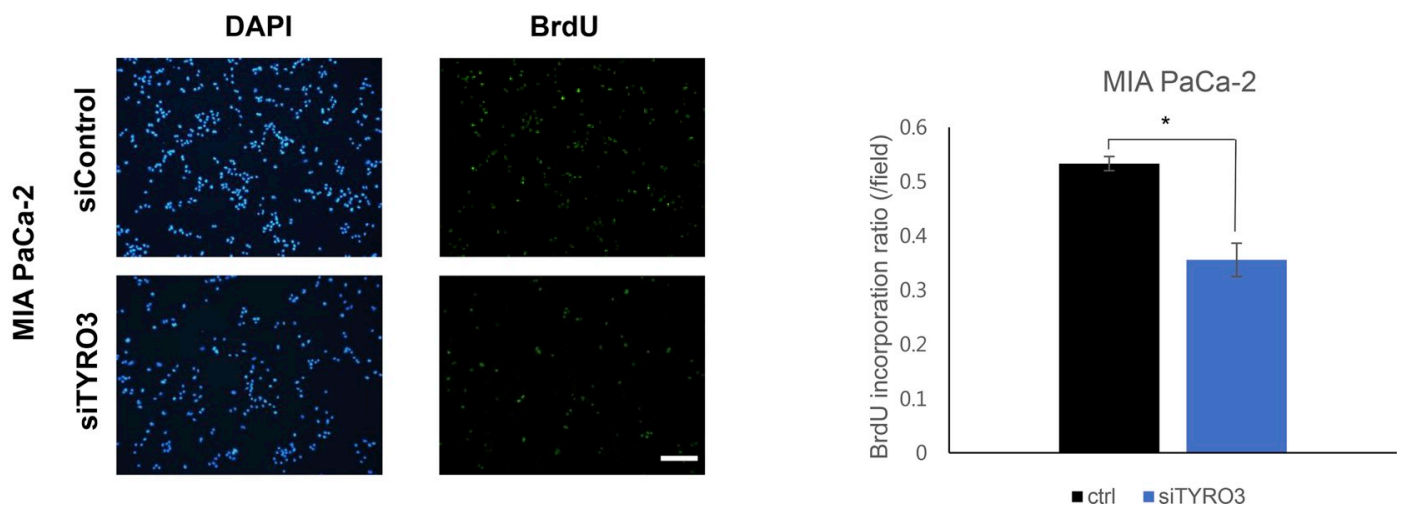

Fig. 1. (continued) 
E
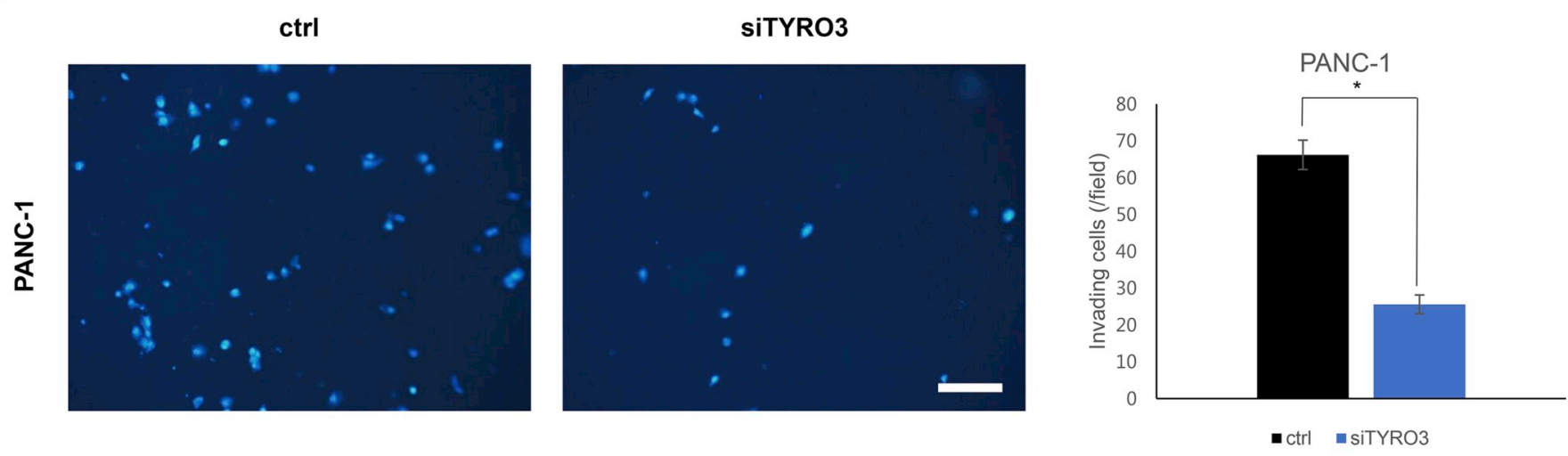

$\mathrm{F}$

ctrl
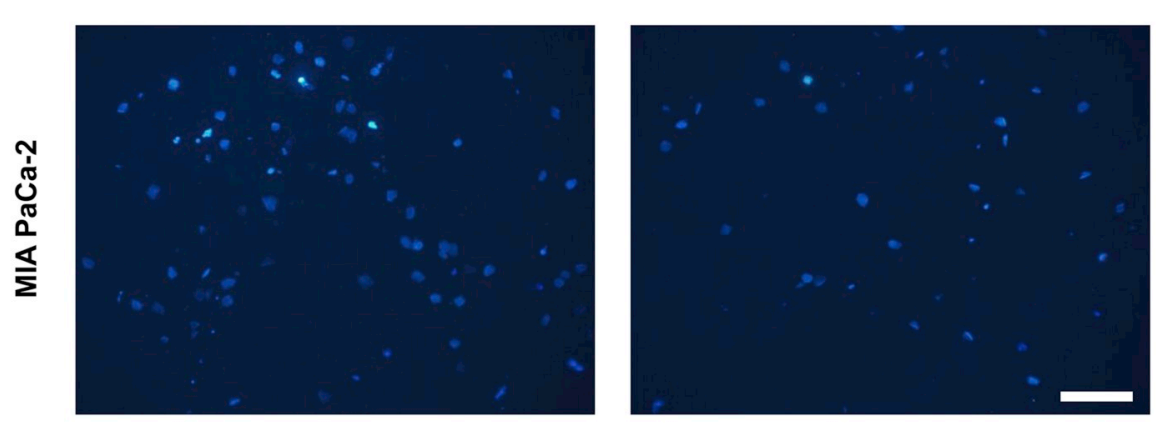

Fig. 1. (continued)

$\mathrm{H}$
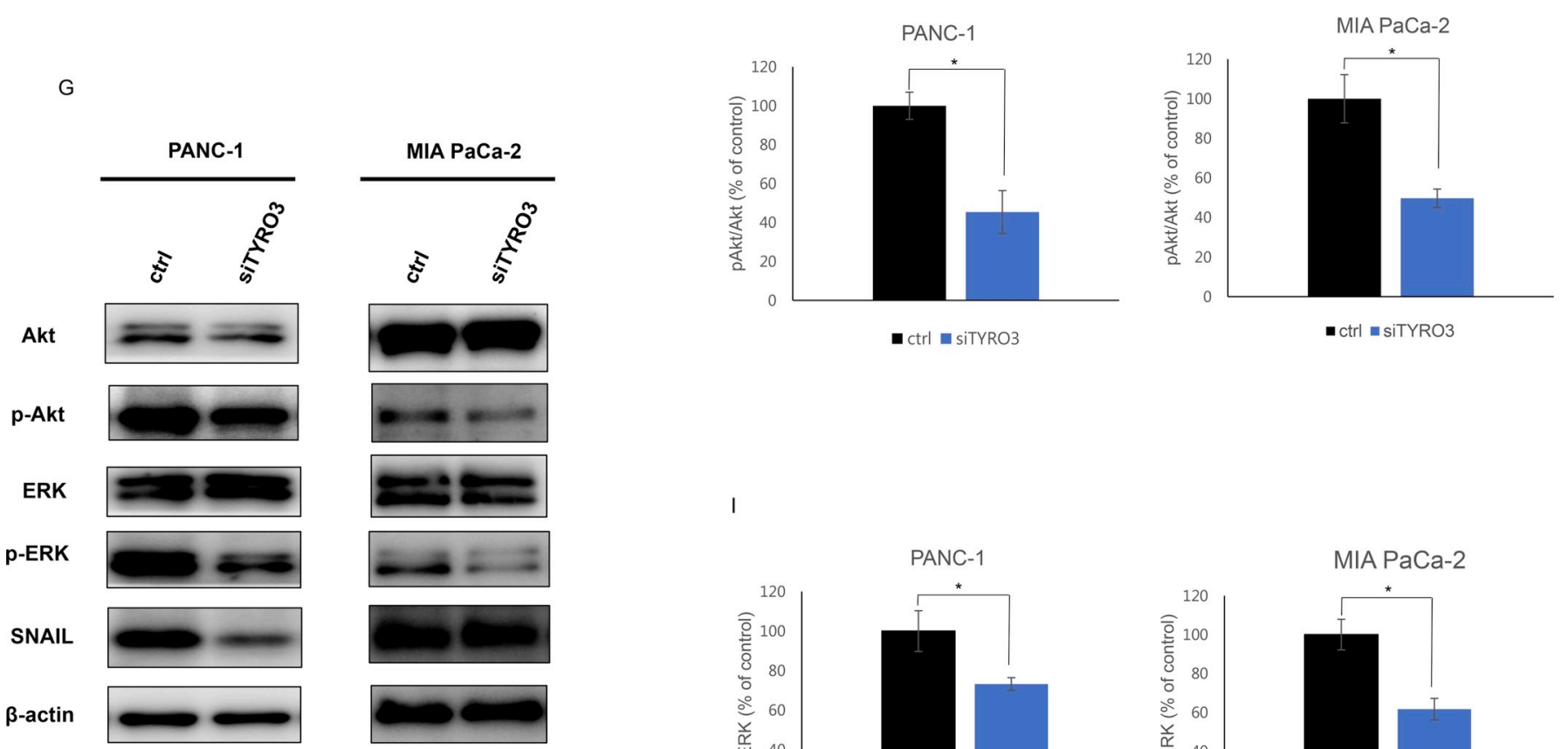
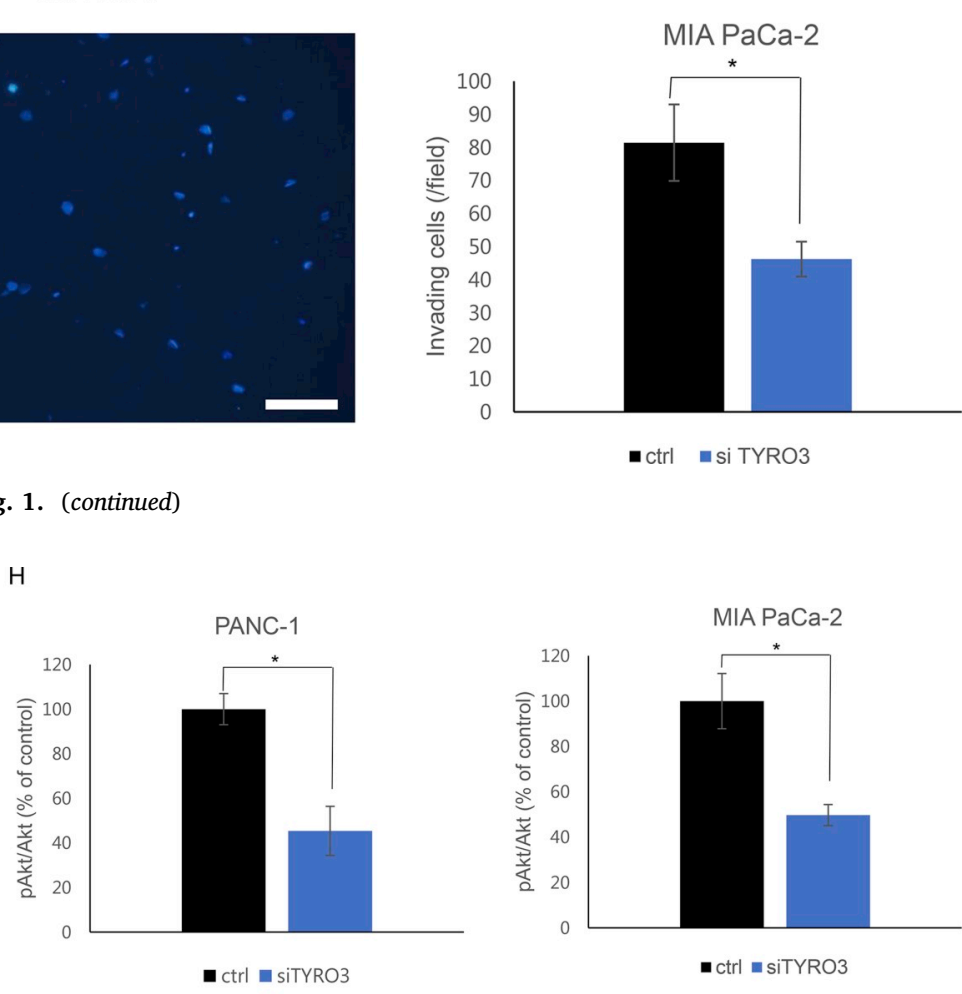

I
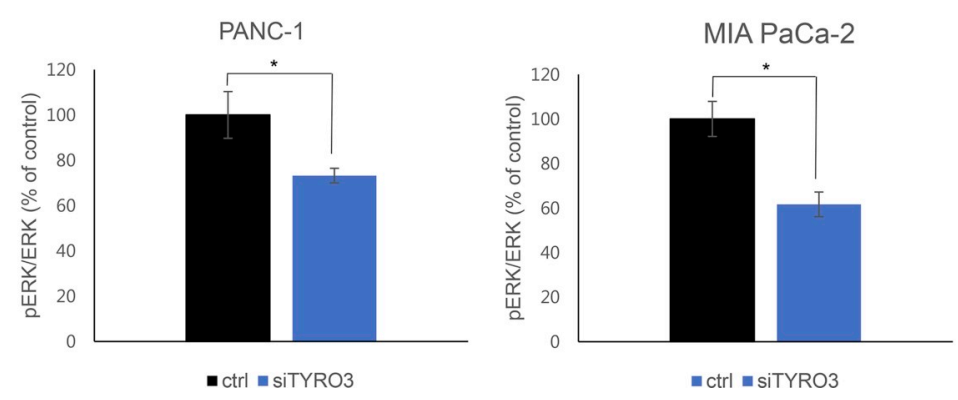

Fig. 1. (continued) 
A

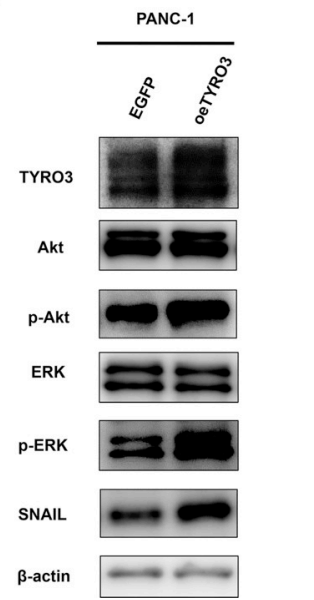

B

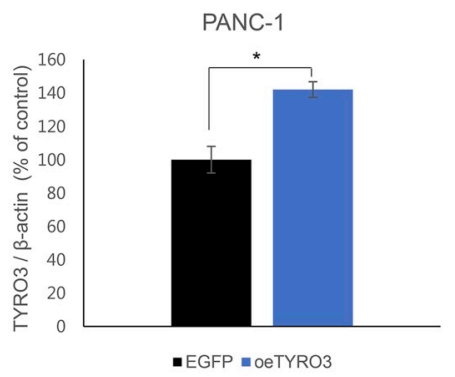

C

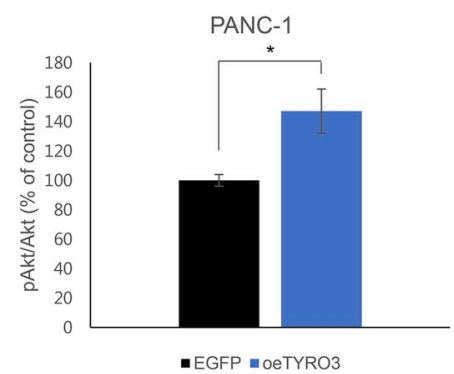

D

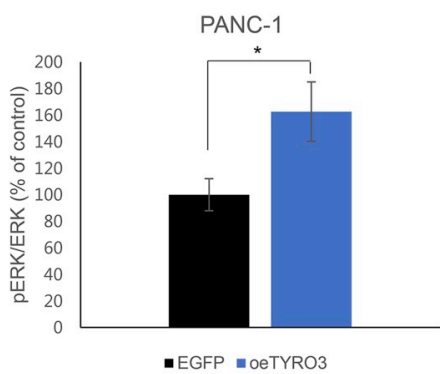

Fig. 2. Effects of TYRO3 overexpression in PC cells on the downstream growth signal pathways and tumor progression. (A)-(D) Western blotting analyses of TYRO3, phospho-Akt/Akt, phospho-ERK/ERK, and SNAIL in TYRO3-overexpressing PANC-1 cells. Western blots were quantified using the Image Quant LAS 4000 mini. (E, F) Confocal photomicrographs showing the altered subcellular localization of phospho-Akt and phospho-ERK in TYRO3-overexpressing PANC-1 cells. Lower panels: EGFP (green), nuclei (blue), and phospho-Akt or phospho-ERK (red). Upper panels: emphasized images of the location of phospho-Akt or phospho-ERK. The arrowheads indicate phospho-Akt or phospho-ERK. Scale bar $=20 \mu \mathrm{m}$. (G) Cells were harvested, fractionated, and analyzed by Western blotting to detect phosphoAkt and phospho-ERK in the membrane and nuclear fractions. Lamin B1 and $\mathrm{Na}^{+} / \mathrm{K}^{+}$ATPase were used as loading controls for the nuclear and membrane fractions, respectively. (H, I) Cell proliferation (H) and invasion (I) in TYRO3-overexpressing PANC-1 cells or control cells were analyzed with the BrdU incorporation assay and the Transwell assay, respectively. The cells were treated with an inhibitor $(20 \mu \mathrm{M} \mathrm{LY}$ or $10 \mu \mathrm{M}$ PD). Scale bar $=200 \mu \mathrm{m}$. LY: LY294002, a PI3K inhibitor. PD: PD98059, a MEK inhibitor. oeTYRO3: TYRO3-overexpressing cells. EGFP: control cells. The data points represent the mean \pm SE of three independent experiments. $* \mathrm{P}<0.05$

TYRO3 in PC cells. TYRO3 knockdown significantly decreased Akt phosphorylation in PC cells (Figs. 1G and 1H). Moreover, TYRO3 depletion significantly suppressed ERK phosphorylation in PC cells, which is another key modulator of signaling in cancer survival and proliferation [31] (Figs. 1G and 1I). Interestingly, TYRO3 silencing decreased SNAIL expression in PANC-1 cells, which is an important inducer of epithelial to mesenchymal transition (EMT) [32] (Fig. 1G). These results associate TYRO3 expression in PC cells with activation of Akt and ERK, which are mediators of tumor growth and proliferation, and induction of SNAIL, a potential EMT signal molecule.

\subsection{Overexpression of TYRO3 in PC cells enhances the downstream growth signaling and tumor progression in vitro}

We performed TYRO3 overexpression experiments in PANC-1 cells to further assess the role of TYRO3 in PC tumor growth. The enhanced TYRO3 expression upregulated Akt and ERK phosphorylation as well as SNAIL expression (Fig. 2A-D). Akt is recruited to the plasma membrane for activation [33], and the activated ERK translocates to the nucleus to phosphorylate transcription factors [34]. We examined the intracellular localization of phospho-Akt and phospho-ERK in TYRO3-overexpressing PC cells via immunocytochemistry using confocal laser microscopy. The overexpression of TYRO3 markedly increased the recruitment of phospho-Akt to the plasma membrane (Fig. 2E) and translocation of phospho-ERK into the nucleus (Fig. 2F). Furthermore, cell fractionation followed by Western blotting using antibodies against total and phosphorylated forms of Akt and ERK confirmed the immunocytochemistry results; both phospho-Akt and phospho-ERK were abundant in the membrane-rich and nuclear fractions, respectively (Fig. 2G). In addition, TYRO3 overexpression significantly promoted the proliferation and invasion of PC cells (Figs. 2H and 2I). Subsequently, we examined the proliferation and invasion in TYRO3-overexpressing PC cells after treatment with LY (a PI3K inhibitor) or PD (a MEK inhibitor) to confirm whether tumor progression, which is promoted by TYRO3, depends on the activation of the PI3K/Akt and MEK/ ERK signaling pathways. Enhancing cell proliferation and invasion by TYRO3 overexpression was abolished after treatment with the inhibitors (Figs. $2 \mathrm{H}$ and 2I), suggesting that TYRO3 promotes the malignant behavior of PC cells via activating growth signaling pathways, such as PI3K/Akt and/or MEK/ERK signaling.

\subsection{TYRO3 silencing mitigates PC tumorigenicity in vivo}

We established a mouse xenograft model using the TYRO3-knockdown PANC-1 clones sh\#C and sh\#D (Fig. 3A) to evaluate the role of TYRO3 in PC tumorigenesis in vivo. The tumor volume was significantly reduced at all time points in the mice subcutaneously injected with the TYRO3-knockdown clones sh\#C and sh\#D compared to the mice injected with a control clone. Five weeks after the injection, both the tumor weight and tumor volume in the mice injected with TYRO3knockdown clones were significantly decreased (Fig. 3B, 3C, and 3D). Furthermore, the levels of TYRO3, phospho-Akt, phospho-ERK, and Ki67 were examined by immunohistochemistry in the xenograft tissue. The expression levels of phospho-Akt and phospho-ERK were positively correlated with that of TYRO3 (Fig. 3E). In addition, the ratios of Ki-67positive tumor cells were significantly lower in the sh\#C and $\mathrm{sh} \# \mathrm{D}$ groups than in the control group (Supplementary Fig. 1). This in vivo mouse xenograft model corroborated the crucial role of TYRO3 in the pancreatic tumor growth shown in our in vitro experiments.

\subsection{Expression of TYRO3 is negatively associated with poor prognosis in patients with $P C$}

We retrospectively analyzed 106 patients with PC (invasive ductal carcinoma, not including IPMN and other nonmalignant tumors) who underwent pancreatic resection to identify the prognostic significance of TYRO3 expression in these patients. We performed immunohistochemical analysis to investigate TYRO3 expression in PC tissue samples obtained from the patients (Fig. 4A), which revealed that TYRO3 expression was positive in $30.2 \%(32 / 106)$ of the samples (Table 1). However, there was no association between TYRO3 expression and other clinicopathological factors (Table 1). We subsequently investigated whether TYRO3 expression was an independent prognostic risk factor for PC. The univariate analysis indicated that histological grade, N stage, CA19-9 level, and TYRO3 expression were risk factors for OS after surgery and disease-specific survival after surgery in 

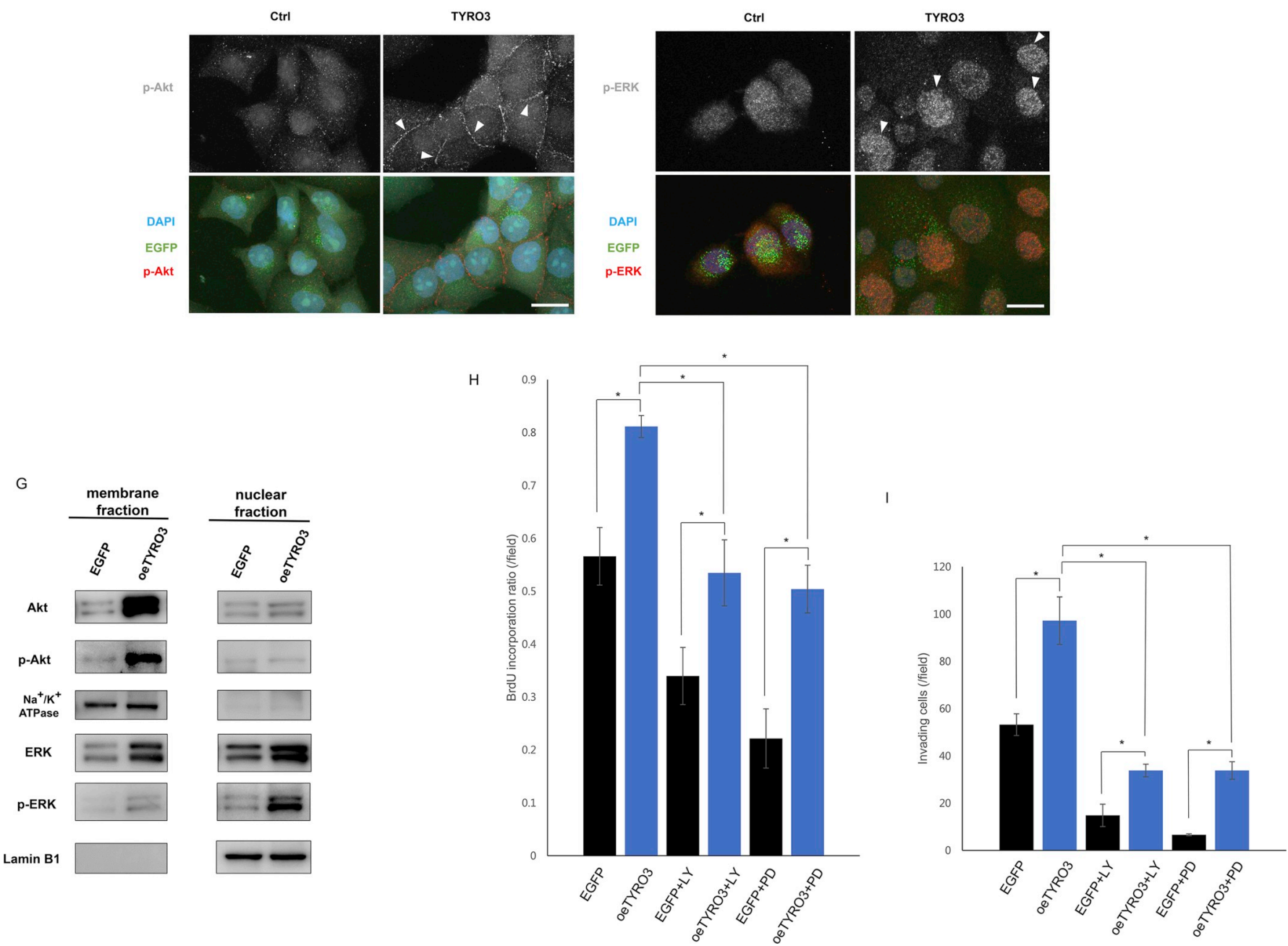

Fig. 2. (continued)

patients with PC (Table 2). The Kaplan-Meier survival analysis demonstrated that the TYRO3-positive patients had significantly worse OS and disease-specific survival compared with the TYRO3-negative patients $(\mathrm{P}=0.0097$ and $\mathrm{P}=0.0081$, respectively). Conversely, TYRO3 positivity was not significantly associated with recurrence-free survival (Fig. 4B, 4C, and 4D). Multivariate analysis using the Cox proportional hazards model revealed that histological grade, N stage, CA19-9 level, and TYRO3 expression were independent prognostic factors for OS in PC patients (Table 3 ). These results suggest that TYRO3 expression is an important prognostic factor for patients with PC.

Moreover, to validate the correlation between TYRO3 and growth signal pathways (PI3K/Akt and MAPK) in clinical samples, we performed immunohistochemical staining for phospho-Akt and phosphoERK in PC samples. The results showed that the PC samples expressed phospho-Akt and phospho-ERK, which were positively correlated with the TYRO3 expression (Fig. 4A).

\section{Discussion}

PC is linked to one of the worst prognoses among all solid tumors [2], and multidisciplinary strategies to diagnose and treat the disease have been evaluated previously [35,36]. However, the current strategies have not yet yielded satisfactory results. Thus, novel therapeutic strategies are required to improve prognosis of patients with PC. In the present study, we focused on members of the relatively new RTK TAM family (i.e., TYRO3, AXL, and MER), which were identified in the 1990s [14-16]. Unlike the other members of the TAM family (i.e., AXL and MER), functional analyses of TYRO3 in tumors, including PC, have not been extensively performed. Thus, we especially aimed to determine TYRO3's role in PC progression.

To the best of our knowledge, the present study is the first to report TYRO3 expression in PC cells. We detected TYRO3 protein expression in all the examined human PC cell lines (i.e., PANC-1, MIA PaCa-2, BxPC-3, AsPC-1, and PK-9). The PC cells used in this study carry some type of mutations in the K-RAS, CDKN2A, TP53, and SMAD4, which might be associated with tumorigenesis [37]. Although members of the TAM family do not have activated mutations contributing to cancer development, it has been suggested that the oncogenic potential of the TAM family is related to aberrant regulation of TAM receptor signaling pathways [24]. In the present study, TYRO3 knockdown in PC cells suppressed cell proliferation and invasion, whereas TYRO3 overexpression enhanced them.

The mechanisms underlying the activation of TAM RTKs, including TYRO3, are unique. The TAM family receptors have an extracellular amino-terminal domain, comprising two immunoglobulin-like domains and two fibronectin type III domains, a single transmembrane domain, 
A

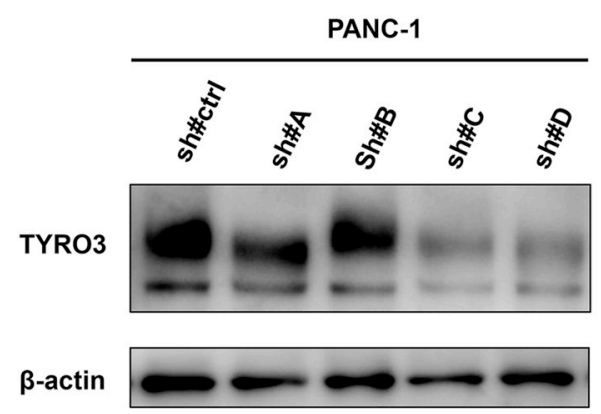

B

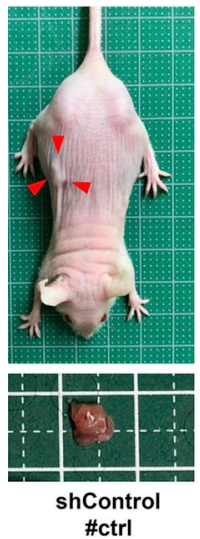

\#ctrl
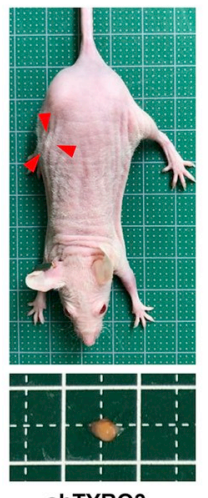

\#C
shTYRO3

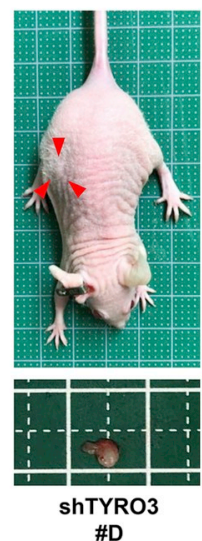

C

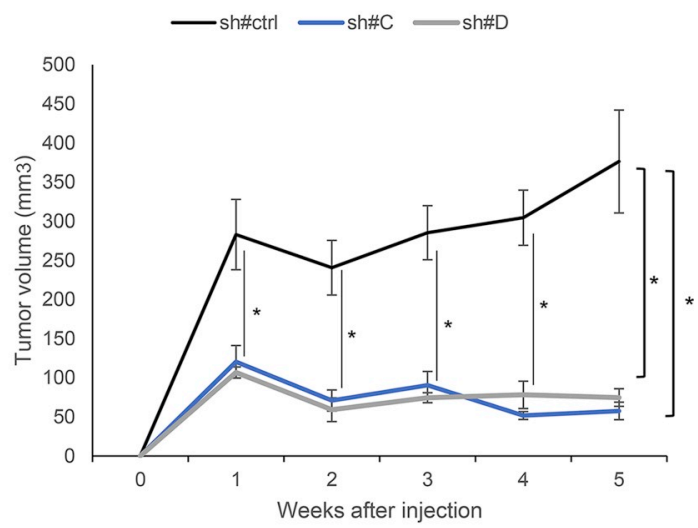

D

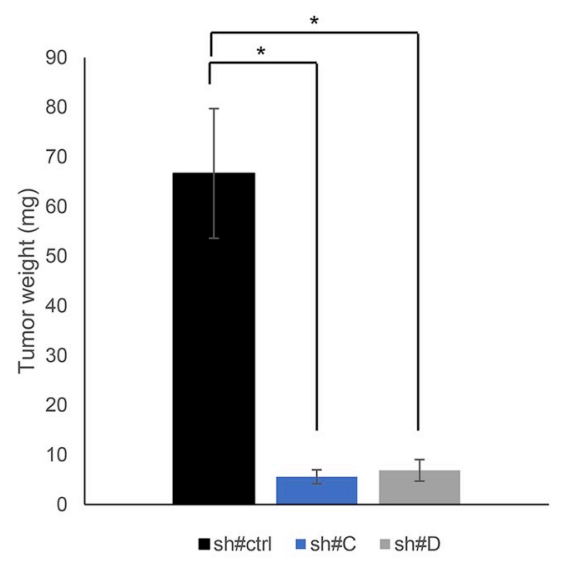

E

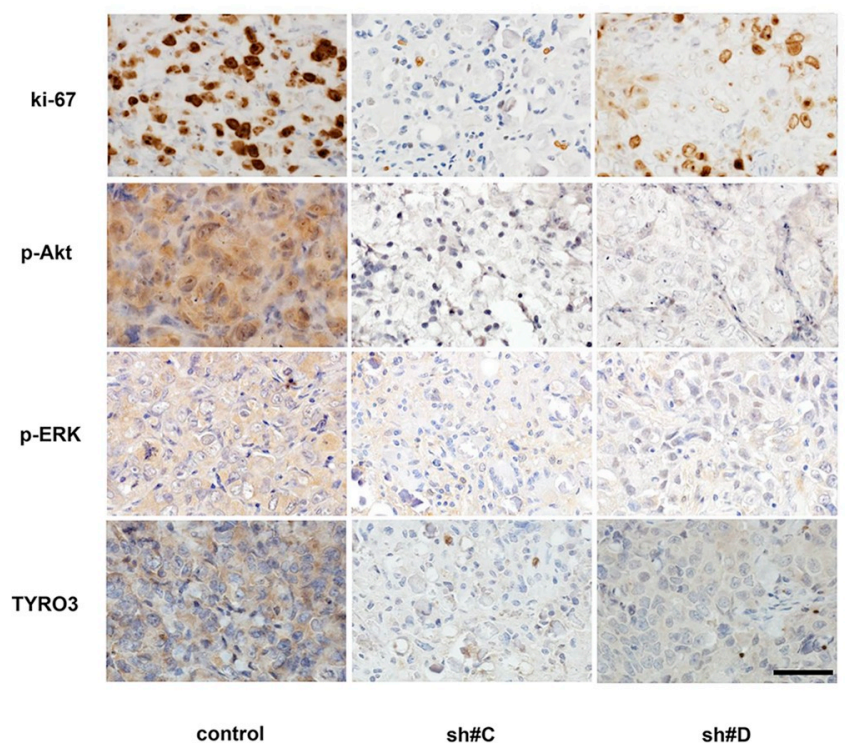

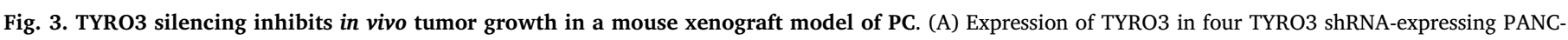

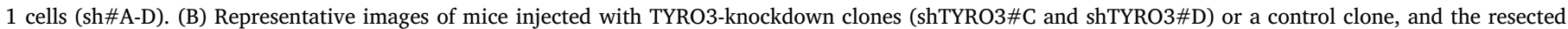

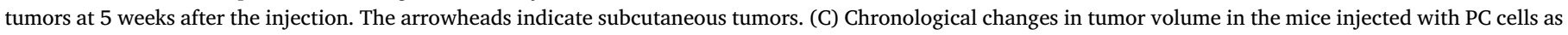

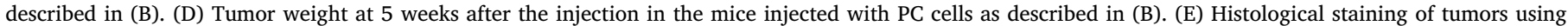
antibodies against Ki-67, phospho-Akt, phospho-ERK, and TYRO3. Scale bar $=50 \mu \mathrm{m}$. The data points represent the mean \pm SE. *P $<0.05$. ctrl, control. 
A

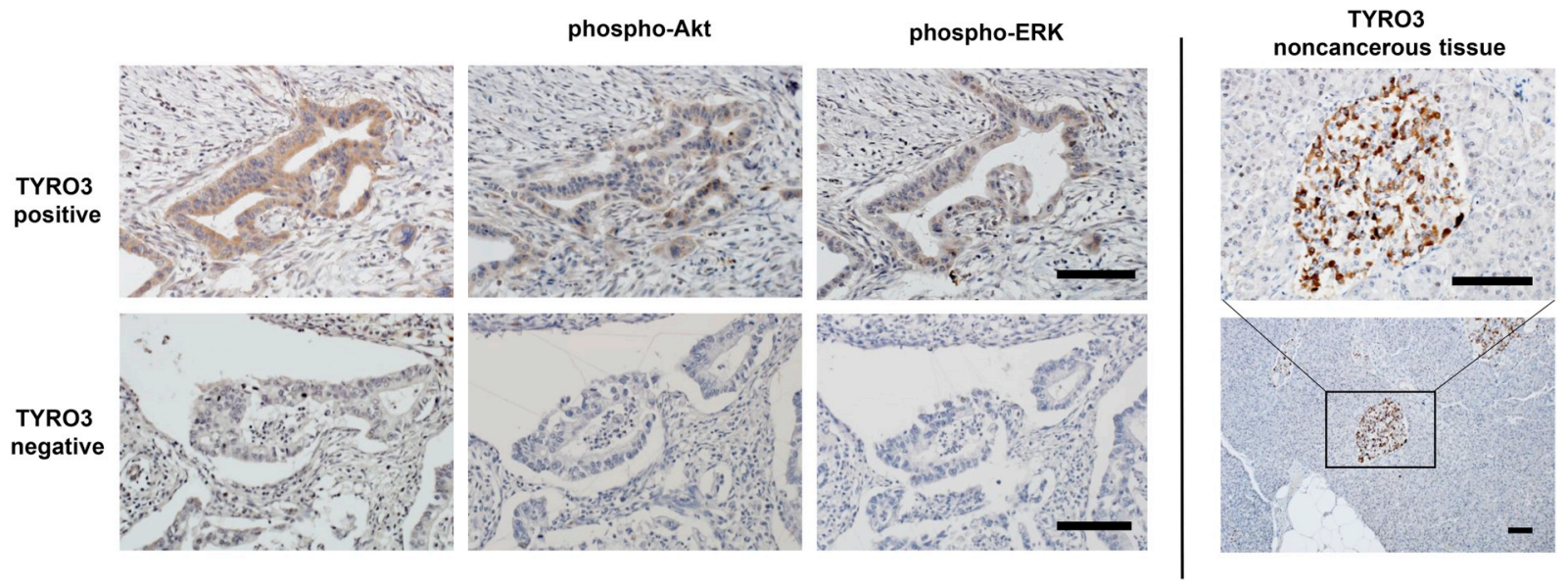

Fig. 4. Negative correlation between TYRO3 expression in PC tissues and prognosis in patients with PC. (A) Representative images of PC tissue samples immunohistochemically stained for TYRO3, phospho-Akt, and phospho-ERK. Image of an islet of Langerhans cells in noncancerous pancreatic tissue used as an internal positive control (Right). Scale bar $=100 \mu \mathrm{m}$. (B)-(D) Survival curves of PC patients with negative $(\mathrm{n}=74)$ and positive ( $\mathrm{n}=32)$ TYRO3 expression.

and an intracellular carboxyl terminus conserved kinase domain featuring the unique KWIAIES sequence. Two major ligands of the TAM family, namely GAS6 [38,39] and PROS1 [40], bind to the receptor with their carboxyl-terminal domain. These ligands also bind to PS with their amino terminus, thus bridging TAM receptors and PS. PROS1 is considered a true ligand of MER and TYRO3; however, it does not bind

B

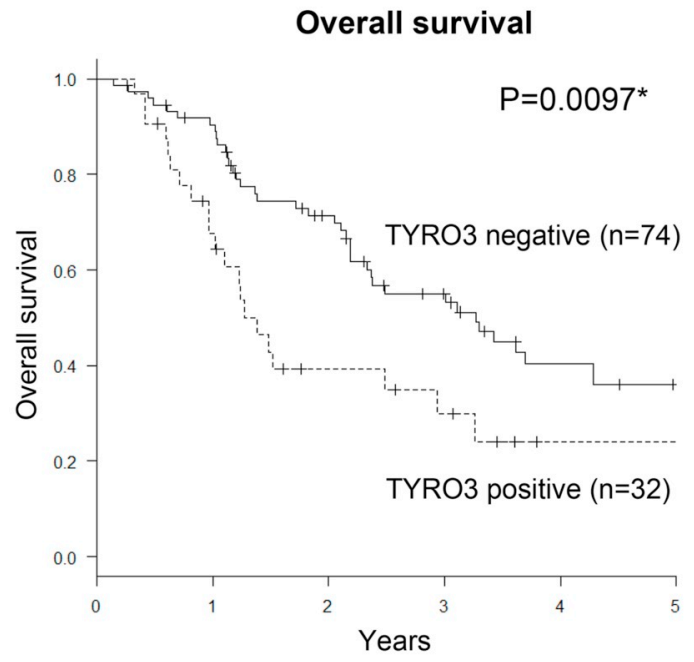

C

Disease specific survival

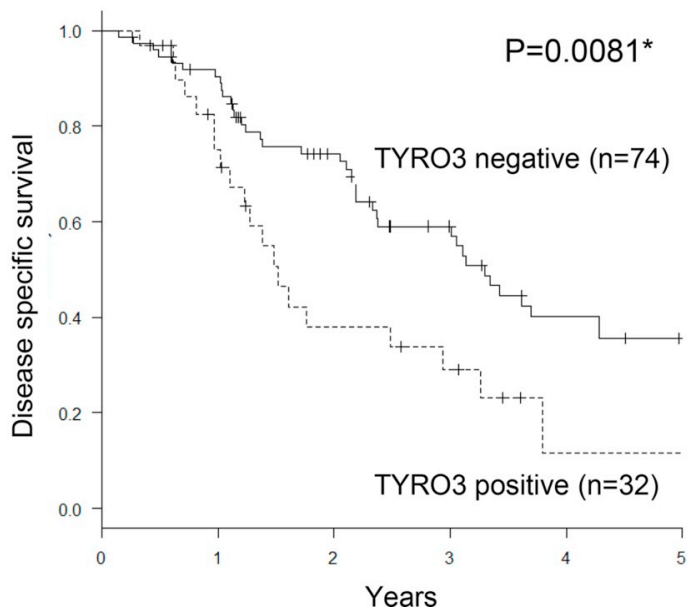

D

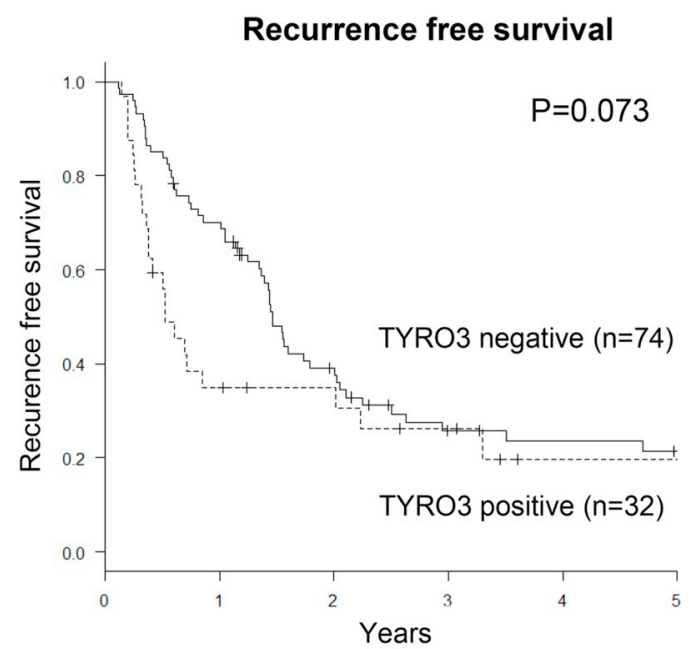

Fig. 4. (continued) 
Table 1

Association between TYRO3 expression and clinicopathological factors.

\begin{tabular}{|c|c|c|c|c|}
\hline \multirow[t]{2}{*}{ parameter } & & \multicolumn{2}{|c|}{ TYRO3 expression } & \multirow[t]{2}{*}{$P$ value } \\
\hline & & $\begin{array}{l}\text { negative } \\
\mathrm{n}=74\end{array}$ & $\begin{array}{l}\text { positive } \\
\mathrm{n}=32\end{array}$ & \\
\hline \multirow[t]{2}{*}{ Age (years) } & $<65$ & 17 & 5 & 0.447 \\
\hline & $\geqq 65$ & 57 & 27 & \\
\hline \multirow[t]{2}{*}{ Sex } & Male & 48 & 20 & 1 \\
\hline & Female & 28 & 12 & \\
\hline \multirow[t]{2}{*}{ BMI } & $<25$ & 63 & 29 & 0.545 \\
\hline & $\geqq 25$ & 11 & 3 & \\
\hline \multirow[t]{2}{*}{ Location } & Head & 43 & 19 & 1 \\
\hline & Body/tail & 31 & 13 & \\
\hline \multirow{2}{*}{$\begin{array}{l}\text { Neoadjuvant } \\
\text { chemotherapy }\end{array}$} & Yes & 9 & 5 & 0.756 \\
\hline & No & 65 & 27 & \\
\hline \multirow[t]{2}{*}{ Adjuvant chemotherapy } & Yes & 43 & 19 & 1 \\
\hline & No & 31 & 13 & \\
\hline Stage & I & 3 & 3 & 0.363 \\
\hline (UICC 7th) & II/III/IV & 71 & 29 & \\
\hline \multirow[t]{3}{*}{ Histlogical type } & well & 33 & 14 & 0.905 \\
\hline & mod/por & 34 & 16 & \\
\hline & others & 7 & 2 & \\
\hline Vascular & negative & 6 & 2 & 1 \\
\hline invasion & positive & 68 & 30 & \\
\hline Lymphatic & negative & 7 & 3 & 1 \\
\hline invasion & positive & 67 & 29 & \\
\hline Neural & negative & 3 & 3 & 0.363 \\
\hline invasion & positive & 71 & 29 & \\
\hline \multirow[t]{2}{*}{ pT } & $\mathrm{I} / \mathrm{II}$ & 5 & 4 & 0.448 \\
\hline & III/IV & 69 & 28 & \\
\hline \multirow[t]{2}{*}{$\mathrm{pN}$} & 0 & 34 & 9 & 0.131 \\
\hline & 1 & 40 & 23 & \\
\hline \multirow[t]{2}{*}{ Margin } & 0 & 61 & 29 & 0.381 \\
\hline & 1 & 13 & 3 & \\
\hline \multirow[t]{2}{*}{ CEA (ng/mL) } & $<5$ & 46 & 16 & 0.286 \\
\hline & $\geqq 5$ & 28 & 16 & \\
\hline \multirow{2}{*}{ CA19-9 (U/mL) } & $<35$ & 26 & 12 & 0.829 \\
\hline & $\geqq 35$ & 48 & 20 & \\
\hline
\end{tabular}

Body mass index; BMI, well/moderately/poorly differentiated; well/mod/por. Pathological T/N; pT/pN.

Carcinoembryonic antigen; CEA, carbohydrate antigen 19-9; CA19-9.

to AXL [17]. PS substantially enhances MER activation, mediated by PROS1 and GAS6, and, to a lesser extent, TYRO3. In contrast, AXL exhibits the highest affinity for GAS6 and may be sufficiently activated via receptor dimerization by GAS6 even in the absence of PS [40]. It has been demonstrated that cancer cells typically present elevated PS levels on the membrane surfaces [41]. Reportedly, PC is under hypoxic conditions primarily because of its hypovascular nature and is subjected to hypoxia-mediated oxidative stress, which enhances PC cell survival [42]. Under oxidative stress [43], PS becomes externalized and changes its form into the oxidized one (i.e., oxidized PS), which demonstrates a relatively higher binding potency for GAS6 [44]. When overexpressed, TYRO3 becomes a homodimer or heterodimer and subsequently autophosphorylates the tyrosine residue within the kinase domain even in the absence of a ligand $[45,46]$. Collectively, TYRO3 may be activated in a ligand-specific manner or via RTK dimerization, followed by autophosphorylation in PC.

The activation of TAM RTKs subsequently stimulates a range of downstream signaling pathways related to tumorigenesis. The PI3K/Akt pathway is one of the most extensively investigated signaling networks in cancer research. Akt, a key mediator of this pathway, is reportedly hyperactivated in various cancer cells by multiple mechanisms including loss of a tumor-suppressor gene PTEN and activation of RAS and growth factor receptors [47]. ERK is an evolutionarily conserved and ubiquitous serine-threonine kinase, which is involved in regulating cell signaling in pathological tissues [48]. Its roles in the MAPK signaling pathway and signal activation by mitogens and mutated RAF or RAS in cancer cells has been studied thoroughly. An early study investigating chimeric MER receptors expressed in NIH3T3 fibroblasts linked both PI3K/Akt and MAPK pathways to MER tyrosine kinase activation [49]. It has also been shown that TYRO3's transforming activity in NIH3T3 fibroblasts is mediated, at least in part, by PI3K/Akt signaling pathway activation [50]. Here, we observed that pretreatment with TYRO3 siRNA suppressed the phosphorylation of Akt and ERK in PC cells. Conversely, we detected an increase in the phosphorylation of these two kinases as well as the recruitment of phospho-Akt to the plasma membrane and translocation of phospho-ERK into the nucleus in TYRO3-overexpressing PANC-1 cells. These observations indicated Akt and ERK activation in TYRO3-overexpressing PC cells. Moreover, the inhibition of PI3K or MEK blocked cell proliferation and invasion in TYRO3-overexpressing PC cells. Interestingly, the expression of SNAIL, an important EMT mediator, was up- and downregulated by the overexpression and knockdown of TYRO3, respectively, in PANC-1 cells. However, TYRO3 knockdown did not affect the expression of SNAIL in MIA PaCa-2 cells. Given that the expression of SNAIL is regulated by several factors [51-53], including TYRO3, the difference in the impact of TYRO3 silencing on the expression of SNAIL between the PANC-1 and MIA PaCa-2 cells may be because of a difference in the regulation of the expression of SNAIL between the two cell lines. Furthermore, we examined the expression of another important EMT transcription factor, SLUG (SNAI2), which also represses the cell adhesion molecule E-cadherin in PC cells. In contrast to SNAIL, SLUG expression was low or not detected in PANC-1 and MIA PaCa-2 cells and was not affected by the overexpression or knockdown of TYRO3 (data not shown). Consistent with the results of the present study, studies have shown that the TAM family induces EMT, stemness, and chemoresistance in other cancer cells [24,54]. It has been shown that AXL and MER regulate some downstream oncogenic signaling pathways (other than the PI3K/Akt and MAPK pathways) by enhancing the expression of anti-apoptotic proteins, such as BCL-2 and BCL-XL [21]; the activation of STATs implicated in tumor cell proliferation and survival $[30,55]$; the activation of cell motility-related proteins, such as FAK1, RHO, and RAC [56-58]; and the activation of the NF-kB pathways [59]. Further investigations will determine the detailed role of TYRO3 in other signaling pathways of oncogenesis, including EMT, in PC cells.

We also corroborated TYRO3's important role in in vivo tumorigenicity using a mouse xenograft model. Five weeks after mice were injected with TYRO3-knockdown PC cells, their tumor volumes were $<50 \%$ of the tumor volumes observed in mice injected with control PC cells. Moreover, tumor weights in mice injected with TYRO3-depleted cells were approximately one-tenth of those in mice injected with control cells. The effects of TYRO3 on tumor growth in vivo were similar to, or more pronounced than, its promoting effects on cell proliferation and invasion in vitro, indicating that TYRO3 is a potent oncogenic driver.

Finally, immunohistochemical analysis using PC specimens obtained from 106 patients with PC revealed that 32 (30.2\%) patients exhibited strong expressions of TYRO3. We confirmed that TYRO3 positivity is an independent prognostic factor of OS in patients with PC. To the best of our knowledge, this is the first study noting a negative correlation between TYRO3 expression and the prognoses of patients with PC.

In conclusion, the present study demonstrates that TYRO3 is constitutively expressed in PC cells and induces tumor progression via Akt and ERK activation. Furthermore, we report for the first time that TYRO3 expression is an independent predictor of prognosis in patients with PC. Determining TYRO3 expression in PC may be useful for predicting patient prognosis, while targeting TYRO3 or its downstream 
Table 2

Survival analyses of prognostic factors.

\begin{tabular}{|c|c|c|c|c|c|c|c|c|c|c|c|}
\hline \multirow[t]{2}{*}{ parameter } & & \multirow[t]{2}{*}{ No. Patient } & \multicolumn{3}{|l|}{ OS } & \multicolumn{3}{|l|}{ DSS } & \multicolumn{3}{|l|}{ RFS } \\
\hline & & & MST (days) & $(95 \% \mathrm{CI})$ & $P$ value & MST (days) & $(95 \% \mathrm{CI})$ & $\mathrm{P}$ value & MST (days) & $(95 \% \mathrm{CI})$ & $\mathrm{P}$ value \\
\hline \multirow[t]{2}{*}{ Age (years) } & $<65$ & 22 & 1134 & 401-NA & 0.88 & 798 & 401-NA & 0.94 & 507 & $133-1718$ & 0.87 \\
\hline & $\geqq 65$ & 84 & 907 & $770-1250$ & & 1098 & $798-1250$ & & 521 & $314-584$ & \\
\hline \multirow[t]{2}{*}{ Sex } & Male & 66 & 798 & 464-1196 & 0.057 & 868 & $541-1222$ & 0.22 & 456 & $227-563$ & 0.22 \\
\hline & Female & 40 & 1566 & 770-NA & & 1190 & 749-NA & & 633 & $295-822$ & \\
\hline \multirow[t]{2}{*}{ BMI } & $<25$ & 92 & 866 & $627-1206$ & 0.45 & 1071 & 749-1222 & 0.46 & 524 & $311-654$ & 0.96 \\
\hline & $\geqq 25$ & 14 & 1321 & 541-NA & & 1321 & 541-NA & & 438 & $150-535$ & \\
\hline \multirow[t]{2}{*}{ Location } & $\overline{\text { Head }}$ & 62 & 1071 & 499-1351 & 0.86 & 1071 & $541-1351$ & 0.48 & 456 & $197-563$ & 0.081 \\
\hline & Body/tail & 44 & 907 & 785-1321 & & 1098 & 798-1566 & & 568 & 383-913 & \\
\hline \multirow[t]{2}{*}{ Neoadjuvant chemotherapy } & Yes & 14 & 1071 & 295-NA & 0.72 & 1206 & 295-NA & 0.52 & 552.5 & 137-NA & 0.52 \\
\hline & No & 92 & 868 & $667-1250$ & & 907 & $770-1250$ & & 498 & $295-570$ & \\
\hline \multirow[t]{2}{*}{ Adjuvant chemotherapy } & Yes & 62 & 1098 & $667-1564$ & 0.35 & 1134 & $785-1351$ & 0.52 & 535 & 314-735 & 0.93 \\
\hline & No & 44 & 799 & $429-1321$ & & 868 & $499-1566$ & & 383 & $212-568$ & \\
\hline Stage & I & 6 & 907 & 150-NA & 0.37 & NA & 798-NA & 0.17 & NA & 222-NA & 0.1 \\
\hline (UICC 7th) & II/III/IV & 100 & 907 & $667-1250$ & & 1098 & $770-1250$ & & 507 & $311-570$ & \\
\hline Histlogical & well & 47 & 1250 & 907-NA & $0.0076^{*}$ & 770 & $439-1190$ & $0.025^{*}$ & 314 & $197-633$ & 0.16 \\
\hline type & mod/por + others & 59 & 667 & 414-907 & & 1222 & 1071-NA & & 563 & $493-815$ & \\
\hline Vascular & negative & 8 & NA & 98-NA & 0.19 & NA & 98-NA & 0.073 & 750 & 89-NA & 0.34 \\
\hline invasion & positive & 98 & 868 & 749-1206 & & 1071 & $770-1222$ & & 507 & $311-570$ & \\
\hline Lymphatic & negative & 10 & NA & 150-NA & 0.2 & NA & NA-NA & $0.029 *$ & 493 & 190-NA & 0.45 \\
\hline invasion & positive & 96 & 868 & $667-1206$ & & 907 & 749-1206 & & 521 & $274-570$ & \\
\hline Neural & negative & 6 & 283 & 150-NA & 0.12 & 406 & $160-\mathrm{NA}$ & 0.85 & NA & 45-NA & 0.47 \\
\hline invasion & positive & 100 & 907 & $785-1250$ & & 1098 & $798-1250$ & & 521 & $314-570$ & \\
\hline \multirow[t]{2}{*}{$\mathrm{pT}$} & $\mathrm{I} / \mathrm{II}$ & 9 & 1321 & 150-NA & 0.61 & 1321 & 406-NA & 0.37 & 381 & 129-NA & 0.48 \\
\hline & III/IV & 97 & 907 & $667-1206$ & & 1098 & $770-1222$ & & 524 & $311-584$ & \\
\hline \multirow[t]{2}{*}{$\mathrm{pN}$} & 0 & 43 & 1566 & 907-NA & $<0.001^{*}$ & NA & 1071-NA & $<0.001^{*}$ & 913 & 524-NA & $<0.001^{*}$ \\
\hline & 1 & 63 & 627 & $450-1098$ & & 644 & $464-1134$ & & 295 & $192-521$ & \\
\hline \multirow[t]{2}{*}{ Margin } & 0 & 90 & 907 & $770-1206$ & 0.67 & 1134 & 798-1321 & 0.72 & 524 & $314-584$ & 0.72 \\
\hline & 1 & 16 & 627 & 371-NA & & 635.5 & 371-NA & & 421.5 & 217-1718 & \\
\hline \multirow[t]{2}{*}{ CEA (ng/mL) } & $<5$ & 62 & 1098 & $770-1564$ & 0.38 & 1146 & $850-1386$ & 0.38 & 528 & $381-731$ & 1 \\
\hline & $\geqq 5$ & 44 & 799 & $450-1250$ & & 798 & $503-1250$ & & 383 & $190-584$ & \\
\hline \multirow[t]{2}{*}{ CA19-9 (U/mL) } & $<35$ & 38 & 1351 & 1071-NA & $0.012^{*}$ & 1386 & 1071-NA & $0.015^{*}$ & 735 & 524-NA & $0.0037^{*}$ \\
\hline & $\geqq 35$ & 68 & 770 & 464-907 & & 785 & 503-1146 & & 311 & $204-524$ & \\
\hline \multirow[t]{2}{*}{ TYRO3 } & positive & 32 & 503 & $353-1071$ & $0.0097^{*}$ & 555 & $401-1071$ & $0.0081^{*}$ & 192 & $132-735$ & 0.073 \\
\hline & negative & 74 & 1196 & 799-1566 & & 1206 & $850-1566$ & & 535 & $456-731$ & \\
\hline
\end{tabular}

Median survival time; MST, confidence interval; CI, overall survival; OS, disease specific survival; DFS, recurrence free survival; RFS.

Body mass index; BMI, well/moderately/poorly differentiated; well/mod/por, pathological T/N; $\mathrm{pT} / \mathrm{pN}$.

Carcinoembryonic antigen; CEA, carbohydrate antigen 19-9; CA19-9, not applicable; NA, statistically significant; *.

Table 3

Multivariate analysis of OS in patients with PC.

\begin{tabular}{lccc}
\hline & HR & $95 \%$ CI & P value \\
\hline Histological type & 0.5673 & $0.3314-0.9712$ & $0.039^{*}$ \\
pNP value & 2.095 & $1.176-3.733$ & $0.012^{*}$ \\
CA19-9 (U/mL) & 1.001 & $1.001-1.001$ & $<0.001^{*}$ \\
positive TYRO3 & 1.762 & $1.013-3.066$ & $0.045^{*}$ \\
\hline
\end{tabular}

Overall survival; OS, pancreatic cancer; PC, hazard ratio; HR, confidence interval; CI.

Pathological N classification; pN, statistically significant; *.

signal pathways may lead to the development of novel therapeutic strategies against PC.

\section{Funding}

This work was partly supported by Japan Society for the Promotion of Science (JSPS) KAKENHI Grant Number 18K16312 (MM), $15 \mathrm{~K} 00816$ (TM), and 18K10992 (TM).

\section{CRediT authorship contribution statement}

Masaki Morimoto: Conceptualization, Writing - original draft, Validation. Yosuke Horikoshi: Methodology, Software, Data curation, Investigation. Kazuhiro Nakaso: Methodology, Visualization. Tatsuyuki Kurashiki: Resources, Data curation. Yoshinori Kitagawa: Resources, Data curation. Takehiko Hanaki: Resources, Data curation.
Teruhisa Sakamoto: Data curation. Soichiro Honjo: Data curation. Yoshihisa Umekita: Supervision, Methodology. Yoshiyuki Fujiwara: Supervision. Tatsuya Matsura: Conceptualization, Project administration, Writing - review \& editing.

\section{Declaration of competing interest}

None.

\section{Acknowledgments}

This study was partly performed using the facilities of the Tottori BioFrontier managed by the Tottori prefecture.

\section{Appendix A. Supplementary data}

Supplementary data to this article can be found online at https:// doi.org/10.1016/j.canlet.2019.11.028.

\section{References}

[1] T. Conroy, J.B. Bachet, A. Ayav, et al., Current standards and new innovative approaches for treatment of pancreatic cancer, Eur. J. Cancer 57 (2016) 10-22.

[2] R.L. Siegel, K.D. Miller, A. Jemal, Cancer statistics, Ca - Cancer J. Clin. 68 (2018) 7-30 2018.

[3] T. Conroy, F. Desseigne, M. Ychou, et al., FOLFIRINOX versus gemcitabine for metastatic pancreatic cancer, N. Engl. J. Med. 364 (2011) 1817-1825.

[4] E. Rozenblum, M. Schutte, M. Goggins, et al., Tumor-suppressive pathways in pancreatic carcinoma, Cancer Res. 57 (1997) 1731-1734. 
[5] Y. Ueda, S. Wang, N. Dumont, J.Y. Yi, Y. Koh, C.L. Arteaga, Overexpression of HER2 (erbB2) in human breast epithelial cells unmasks transforming growth factor betainduced cell motility, J. Biol. Chem. 279 (2004) 24505-24513.

[6] A. Bera, S. Zhao, L. Cao, P.J. Chiao, J.W. Freeman, Oncogenic K-Ras and loss of Smad4 mediate invasion by activating an EGFR/NF-kappaB Axis that induces expression of MMP9 and uPA in human pancreas progenitor cells, PLoS One 8 (2013) e82282.

[7] Y.J. Kim, K. Jung, D.S. Baek, S.S. Hong, Y.S. Kim, Co-targeting of EGF receptor and neuropilin-1 overcomes cetuximab resistance in pancreatic ductal adenocarcinoma with integrin beta1-driven Src-Akt bypass signaling, Oncogene 36 (2017) $2543-2552$

[8] M.J. Moore, D. Goldstein, J. Hamm, et al., Erlotinib plus gemcitabine compared with gemcitabine alone in patients with advanced pancreatic cancer: a phase III trial of the National Cancer Institute of Canada Clinical Trials Group, J. Clin. Oncol. 25 (2007) 1960-1966.

[9] G. Middleton, D.H. Palmer, W. Greenhalf, et al., Vandetanib plus gemcitabine versus placebo plus gemcitabine in locally advanced or metastatic pancreatic carcinoma (ViP): a prospective, randomised, double-blind, multicentre phase 2 trial, Lancet Oncol. 18 (2017) 486-499.

[10] A. Amedei, E. Niccolai, M. Benagiano, et al., Ex vivo analysis of pancreatic cancerinfiltrating T lymphocytes reveals that ENO-specific Tregs accumulate in tumor tissue and inhibit Th1/Th17 effector cell functions, Cancer Immunol. Immunother. 62 (2013) 1249-1260.

[11] B. Farrow, Y. Sugiyama, A. Chen, E. Uffort, W. Nealon, B. Mark Evers, Inflammatory mechanisms contributing to pancreatic cancer development, Ann. Surg. 239 (2004) 763-769 discussion 769-771.

[12] A. Masamune, T. Watanabe, K. Kikuta, T. Shimosegawa, Roles of pancreatic stellate cells in pancreatic inflammation and fibrosis, Clin. Gastroenterol. Hepatol. 7 (2009) S48-S54.

[13] F. Lapraz, E. Rottinger, V. Duboc, et al., RTK and TGF-beta signaling pathways genes in the sea urchin genome, Dev. Biol. 300 (2006) 132-152.

[14] C. Lai, M. Gore, G. Lemke, Structure, expression, and activity of Tyro 3, a neural adhesion-related receptor tyrosine kinase, Oncogene 9 (1994) 2567-2578.

[15] J.P. O'Bryan, R.A. Frye, P.C. Cogswell, et al., axl, a transforming gene isolated from primary human myeloid leukemia cells, encodes a novel receptor tyrosine kinase, Mol. Cell. Biol. 11 (1991) 5016-5031.

[16] D.K. Graham, T.L. Dawson, D.L. Mullaney, H.R. Snodgrass, H.S. Earp, Cloning and mRNA expression analysis of a novel human protooncogene, c-mer, Cell Growth Differ. 5 (1994) 647-657.

[17] D.K. Graham, D. DeRyckere, K.D. Davies, H.S. Earp, The TAM family: phosphatidylserine sensing receptor tyrosine kinases gone awry in cancer, Nat. Rev. Cancer 14 (2014) 769-785.

[18] N.B. Caberoy, G. Alvarado, W. Li, Tubby regulates microglial phagocytosis through MerTK, J. Neuroimmunol. 252 (2012) 40-48.

[19] N.B. Caberoy, G. Alvarado, J.L. Bigcas, W. Li, Galectin-3 is a new MerTK-specific eat-me signal, J. Cell. Physiol. 227 (2012) 401-407.

[20] P. Vajkoczy, P. Knyazev, A. Kunkel, et al., Dominant-negative inhibition of the Axl receptor tyrosine kinase suppresses brain tumor cell growth and invasion and prolongs survival, Proc. Natl. Acad. Sci. U. S. A. 103 (2006) 5799-5804.

[21] R.M. Linger, R.A. Cohen, C.T. Cummings, et al., Mer or Axl receptor tyrosine kinase inhibition promotes apoptosis, blocks growth and enhances chemosensitivity of human non-small cell lung cancer, Oncogene 32 (2013) 3420-3431.

[22] K.F. Ludwig, W. Du, N.B. Sorrelle, et al., Small-molecule inhibition of axl targets tumor immune suppression and enhances chemotherapy in pancreatic cancer, Cancer Res. 78 (2018) 246-255.

[23] L. Sinik, K.A. Minson, J.J. Tentler, et al., Inhibition of MERTK promotes suppression of tumor growth in BRAF mutant and BRAF wild-type melanoma, Mol. Cancer Ther. 18 (2019) 278-288.

[24] C.W. Chien, P.C. Hou, H.C. Wu, et al., Targeting TYRO3 inhibits epithelial-mesenchymal transition and increases drug sensitivity in colon cancer, Oncogene 35 (2016) 5872-5881.

[25] E. Avilla, V. Guarino, C. Visciano, et al., Activation of TYRO3/AXL tyrosine kinase receptors in thyroid cancer, Cancer Res. 71 (2011) 1792-1804.

[26] S. Zhu, H. Wurdak, Y. Wang, et al., A genomic screen identifies TYRO3 as a MITF regulator in melanoma, Proc. Natl. Acad. Sci. U. S. A. 106 (2009) 17025-17030.

[27] T. Yamanaka, Y. Horikoshi, Y. Sugiyama, et al., Mammalian Lgl forms a protein complex with PAR- 6 and aPKC independently of PAR-3 to regulate epithelial cell polarity, Curr. Biol. 13 (2003) 734-743.

[28] T. Hanaki, Y. Horikoshi, K. Nakaso, et al., Nicotine enhances the malignant potential of human pancreatic cancer cells via activation of atypical protein kinase C, Biochim. Biophys. Acta 1860 (2016) 2404-2415.

[29] Y. Shiozawa, E.A. Pedersen, L.R. Patel, et al., GAS6/AXL axis regulates prostate cancer invasion, proliferation, and survival in the bone marrow niche, Neoplasia 12 (2010) 116-127.

[30] A.B. Lee-Sherick, K.M. Eisenman, S. Sather, et al., Aberrant Mer receptor tyrosine kinase expression contributes to leukemogenesis in acute myeloid leukemia, Oncogene 32 (2013) 5359-5368.

[31] A. Herrero, A. Pinto, P. Colon-Bolea, et al., Small molecule inhibition of ERK dimerization prevents tumorigenesis by RAS-ERK pathway oncogenes, Cancer Cell 28 (2015) 170-182.

[32] B. Boyer, A.M. Valles, N. Edme, Induction and regulation of epithelial-mesenchymal transitions, Biochem. Pharmacol. 60 (2000) 1091-1099.

[33] Y. Zhao, Y. Lin, H. Zhang, et al., Ubl4A is required for insulin-induced Akt plasma membrane translocation through promotion of Arp2/3-dependent actin branching, Proc. Natl. Acad. Sci. U. S. A. 112 (2015) 9644-9649.

[34] F. Liu, X. Yang, M. Geng, M. Huang, Targeting ERK, an Achilles' Heel of the MAPK pathway, in cancer therapy, Acta Pharm. Sin. B 8 (2018) 552-562.

[35] J. Taieb, A.L. Pointet, J.L. Van Laethem, et al., What treatment in 2017 for inoperable pancreatic cancers? Ann. Oncol. 28 (2017) 1473-1483.

[36] D. Ansari, A. Gustafsson, R. Andersson, Update on the management of pancreatic cancer: surgery is not enough, World J. Gastroenterol. 21 (2015) 3157-3165.

[37] E.L. Deer, J. Gonzalez-Hernandez, J.D. Coursen, et al., Phenotype and genotype of pancreatic cancer cell lines, Pancreas 39 (2010) 425-435.

[38] T.N. Stitt, G. Conn, M. Gore, et al., The anticoagulation factor protein S and its relative, Gas6, are ligands for the Tyro 3/Axl family of receptor tyrosine kinases, Cell 80 (1995) 661-670.

[39] B.C. Varnum, C. Young, G. Elliott, et al., Axl receptor tyrosine kinase stimulated by the vitamin K-dependent protein encoded by growth-arrest-specific gene 6, Nature 373 (1995) 623-626.

[40] W.I. Tsou, K.Q. Nguyen, D.A. Calarese, et al., Receptor tyrosine kinases, TYRO3, AXL, and MER, demonstrate distinct patterns and complex regulation of ligandinduced activation, J. Biol. Chem. 289 (2014) 25750-25763.

[41] T. Utsugi, A.J. Schroit, J. Connor, C.D. Bucana, I.J. Fidler, Elevated expression of phosphatidylserine in the outer membrane leaflet of human tumor cells and recognition by activated human blood monocytes, Cancer Res. 51 (1991) 3062-3066.

[42] S. Joshi, S. Kumar, M.P. Ponnusamy, S.K. Batra, Hypoxia-induced oxidative stress promotes MUC4 degradation via autophagy to enhance pancreatic cancer cells survival, Oncogene 35 (2016) 5882-5892.

[43] T. Matsura, Oxidized phosphatidylserine: production and bioactivities, Yonago Acta Med. 57 (2014) 119-127.

[44] V.A. Tyurin, K. Balasubramanian, D. Winnica, et al., Oxidatively modified phosphatidylserines on the surface of apoptotic cells are essential phagocytic 'eat-me signals: cleavage and inhibition of phagocytosis by Lp-PLA2, Cell Death Differ. 21 (2014) 825-835.

[45] J. Schlessinger, Cell signaling by receptor tyrosine kinases, Cell 103 (2000) 211-225.

[46] I.C. Taylor, S. Roy, H.E. Varmus, Overexpression of the Sky receptor tyrosine kinase at the cell surface or in the cytoplasm results in ligand-independent activation, Oncogene 11 (1995) 2619-2626.

[47] Q. Wang, X. Chen, N. Hay, Akt as a target for cancer therapy: more is not always better (lessons from studies in mice), Br. J. Canc. 117 (2017) 159-163.

[48] Y. Pylayeva-Gupta, E. Grabocka, D. Bar-Sagi, RAS oncogenes: weaving a tumorigenic web, Nat. Rev. Cancer 11 (2011) 761-774.

[49] L. Ling, H.J. Kung, Mitogenic signals and transforming potential of Nyk, a newly identified neural cell adhesion molecule-related receptor tyrosine kinase, Mol. Cell. Biol. 15 (1995) 6582-6592.

[50] Z. Lan, H. Wu, W. Li, et al., Transforming activity of receptor tyrosine kinase tyro3 is mediated, at least in part, by the PI3 kinase-signaling pathway, Blood 95 (2000) $633-638$

[51] P. Dong, Y. Xiong, H. Watari, et al., MiR-137 and miR-34a directly target Snail and inhibit EMT, invasion and sphere-forming ability of ovarian cancer cells, J. Exp. Clin. Cancer Res. 35 (2016) 132.

[52] Q. Ji, X. Hao, M. Zhang, et al., MicroRNA miR-34 inhibits human pancreatic cance tumor-initiating cells, PLoS One 4 (2009) e6816.

[53] K. Horiguchi, T. Shirakihara, A. Nakano, T. Imamura, K. Miyazono, M. Saitoh, Role of Ras signaling in the induction of snail by transforming growth factor-beta, J. Biol. Chem. 284 (2009) 245-253.

[54] M.K. Asiedu, F.D. Beauchamp-Perez, J.N. Ingle, M.D. Behrens, D.C. Radisky, K.L. Knutson, AXL induces epithelial-to-mesenchymal transition and regulates the function of breast cancer stem cells, Oncogene 33 (2014) 1316-1324.

[55] K.M. Giles, F.C. Kalinowski, P.A. Candy, et al., Axl mediates acquired resistance of head and neck cancer cells to the epidermal growth factor receptor inhibitor erlotinib, Mol. Cancer Ther. 12 (2013) 2541-2558.

[56] A.E. Rogers, J.P. Le, S. Sather, et al., Mer receptor tyrosine kinase inhibition impedes glioblastoma multiforme migration and alters cellular morphology, Oncogene 31 (2012) 4171-4181.

[57] N.P. Mahajan, H.S. Earp, An SH2 domain-dependent, phosphotyrosine-independent interaction between Vav1 and the Mer receptor tyrosine kinase: a mechanism for localizing guanine nucleotide-exchange factor action, J. Biol. Chem. 278 (2003) 42596-42603.

[58] M.P. Allen, D.A. Linseman, H. Udo, et al., Novel mechanism for gonadotropin-releasing hormone neuronal migration involving Gas6/Ark signaling to p38 mitogenactivated protein kinase, Mol. Cell. Biol. 22 (2002) 599-613.

[59] K.Y. Tai, Y.S. Shieh, C.S. Lee, S.G. Shiah, C.W. Wu, Axl promotes cell invasion by inducing MMP-9 activity through activation of NF-kappaB and Brg-1, Oncogene 27 (2008) 4044-4055 